\title{
Schubert Calculus on a Grassmann Algebra*
}

\author{
Letterio Gatto, Taíse Santiago
}

\begin{abstract}
The (classical, small quantum, equivariant) cohomology ring of the grassmannian $G(k, n)$ is generated by certain derivations operating on an exterior algebra of a free module of rank $n$ (Schubert Calculus on a Grassmann Algebra). Our main result gives, in a unified way, a presentation of all such cohomology rings in terms of generators and relations. It also provides, by results of Laksov and Thorup ([8] and [9]), a presentation of the universal splitting algebra of a monic polynomial of degree $n$ into the product of two monic polynomials, one of degree $k$.
\end{abstract}

\section{Introduction}

In the paper [4 one shows that the cohomology ring of the complex grassmannian $G(k, n)$, parametrizing $k$-dimensional subspaces of $\mathbb{C}^{n}$, can be realized as a commutative ring of endomorphism of the $k^{\text {th }}$ exterior power of a free $\mathbb{Z}$-module $M$ of rank $n$. Such a result was achieved by studying a natural Hasse-Schmidt derivation on the exterior algebra of $M$; Laksov and Thorup ([8] and [9]) generalized it to the more interesting situation regarding the cohomology of Grassmann bundles. Their point of view is quite different, as it is based on the fact that the $k^{t h}$-exterior power of a free $A$-module of rank $n$ can be endowed with a natural module structure over the ring of symmetric polynomials (with $A$-coefficients): this leads to a beautiful and natural description of the cohomology of $G(k, E)$, the Grassmann bundle of $k$-dimensional subspaces in the fibers of a vector bundle $E$, in terms of the universal splitting algebra of a certain monic polynomial $\mathrm{p}$ (encoding the Chern classes of $E$ ) into the product of two monic polynomials, one of degree $k$ (Cf. remark 3.11).

The main goal of this paper is to generalize [4 via a translation of Laksov and Thorup's formalism into the language of derivations. A derivation on $\wedge M$, the exterior algebra of a module $M$ over a commutative ring with unit, is a sequence $D:=$ $\left(D_{0}, D_{1}, \ldots\right)$ of endomorphisms, such that the $h^{\text {th }}$ order Leibniz's rule:

$$
D_{h}(\alpha \wedge \beta)=\sum_{\substack{h_{1}+h_{2}=h \\ h_{i} \geq 0}} D_{h_{1}} \alpha \wedge D_{h_{2}} \beta,
$$

*Work partially sponsored by PRIN "Geometria sulle Varietà Algebriche" (Coordinatore A. Verra), INDAM-GNSAGA and ScuDo, Politecnico di Torino. 
holds for each $h \geq 0$ and each $\alpha, \beta \in \bigwedge M$ (see 2.2). In [12], any such a derivation is called a Schubert Calculus on a Grassmann Algebra. The terminology is motivated by the fact that if one takes $M$ to be a finite free module over a graded commutative $\mathbb{Z}$-algebra of characteristic 0 , there is a canonical derivation on $\bigwedge M$ (generalizing that studied in (4); see Section 3) describing, within a unified framework, different kind of cohomology theories on complex grassmannian varieties, such as, e.g., the classical, the small quantum or the equivariant one. Working on the exterior algebra, instead of on a single exterior power, many formal manipulations get easier: as an example we offer Theorem 4.8, the main result of this paper, that consists in a simple formula giving, in a unified way, the presentation of the classical, small quantum and equivariant cohomology ring of the complex grassmannian $G(k, n)$. In fact, the (classical, small quantum, equivariant) cohomology ring of all the grassmannians $G(k, n), 1 \leq k \leq n$, are quotient of a same commutative ring of endomorphisms of the exterior algebra of a free module of rank $n$ (see Sect. 3.7). As the latter is generated by derivations, the (classical, small quantum, equivariant) Schubert calculus on $G(k, n)$ can be reduced to that, much easier, on $G(1, n)=\mathbb{P}^{n-1}$ (as in [4; see also [5]). Our best application of such a philosophy regards an elementary description, as in [6] (see also [12), of the equivariant Schubert calculus on a grassmannian acted on by a torus with isolated fixed locus, recovering, in particular, the case studied in [7] (see also [10]).

Acknowledgment. The first author wants to thank the warm ospitality of the STID of Menton, Université de Nice, Sophia-Antipolis, notably that of its chairmain, Guy Choisnet, where most part of this paper, originated from [4] and 12, has been written. The current exposition has been deeply influenced by the work of D. Laksov and A. Thorup on related subjects ([8], [9, [10]) and by many conversations the authors had with the former, to whom they want to address a warm feeling of gratitude. We also thank I. Vainsencher for some key suggestions as well as the Referee for his valuable and (especially) patient remarks.

\section{Derivations on Exterior Algebras}

2.1 Let $M$ be an $A$-module, $A[[t]]$ be the ring of formal power series in an indeterminate $t$ over $A$ and $\bigwedge M[[t]]:=(\bigwedge M)[[t]]$ be the $A[[t]]$-module of formal power series with coefficients in $\bigwedge M=\bigoplus_{k \geq 0} \Lambda^{k} M$, the exterior algebra of $M$. The former gets a structure of $A[[t]]$-algebra by setting $\sum_{i \geq 0} \alpha_{i} t^{i} \wedge \sum_{j \geq 0} \beta_{j} t^{j}=\sum_{h \geq 0} \sum_{i+j=h}\left(\alpha_{i} \wedge \beta_{j}\right) t^{h}$.

2.2 An $A$-module homomorphism $D_{t}: \bigwedge M \rightarrow \bigwedge M[[t]]$ is said to be a derivation on $\bigwedge M$ if it is an $A$-algebra homomorphism, i.e. if for each $\alpha, \beta \in \wedge M$ :

$$
D_{t}(\alpha \wedge \beta)=D_{t} \alpha \wedge D_{t} \beta
$$

The algebra homomorphism $D_{t}$ can be written as a formal power series $\sum_{i>0} D_{i} t^{i}$, with coefficients in the $A$-algebra $\operatorname{End}_{A}(\bigwedge M)$. Denote by $D$ the sequence $\left(D_{0}, D_{1}, \ldots\right)$ of the coefficients of $D_{t}$. Equation (2) implies that for each $h \geq 0$, the $A$-endomorphism $D_{h}$ of $\bigwedge M$ satisfies the $h^{t h}$-order Leibniz rule (11), got by expanding both sides of (2) and equating the coefficients of $t^{h}$ occurring on both sides. 
2.3 Let $\jmath: \operatorname{Hom}_{A}(\bigwedge M, \bigwedge M[[t]]) \rightarrow \operatorname{End}_{A}(\bigwedge M[[t]])$ be the natural map sending any $\Psi_{t}=\sum_{i \geq 0} \psi_{i} t^{i} \in \operatorname{Hom}_{A}(\bigwedge M, \bigwedge M[[t]])$ to the endomorphism $\jmath(\Psi)$ of $\bigwedge M[[t]]$, defined, on each $\sum_{i \geq 0} \alpha_{i} t^{i} \in \bigwedge M[[t]]$, as:

$$
\jmath(\Psi)\left(\sum_{i \geq 0} \alpha_{i} t^{i}\right)=\sum_{i \geq 0} \Psi\left(\alpha_{i}\right) \cdot t^{i}=\sum_{h \geq 0}\left(\sum_{i+j=h} \psi_{i}\left(\alpha_{j}\right)\right) t^{h} .
$$

If $D_{t}$ is a derivation, then $\jmath\left(D_{t}\right)$ is itself an $A[[t]]$-algebra endomorphism of $\bigwedge M[[t]]$. In fact it is obviously an $A[[t]]$-module endomorphism and, moreover:

$$
\begin{aligned}
\jmath\left(D_{t}\right)\left(\sum_{i \geq 0} \alpha_{i} t^{i} \wedge \sum_{j \geq 0} \beta_{j} t^{j}\right) & =\jmath\left(D_{t}\right) \sum_{h \geq 0}\left(\sum_{i+j=h} \alpha_{i} \wedge \beta_{j}\right) t^{h}= \\
=\sum_{h \geq 0}\left(\sum_{i+j=h} D_{t}\left(\alpha_{i} \wedge \beta_{j}\right)\right) t^{h} & =\sum_{h \geq 0}\left(\sum_{i+j=h} D_{t} \alpha_{i} \wedge D_{t} \beta_{j}\right) t^{h}= \\
= & \sum_{i \geq 0} D_{t} \alpha_{i} \cdot t^{i} \wedge \sum_{j \geq 0} D_{t} \beta_{j} \cdot t^{j}=\jmath\left(D_{t}\right) \sum_{i \geq 0} \alpha_{i} \cdot t^{i} \wedge \jmath\left(D_{t}\right) \sum_{j \geq 0} \beta_{j} \cdot t^{j} .
\end{aligned}
$$

2.4 For each pair $D_{t}, D_{t}^{\prime} \in H_{o m}(\bigwedge M, \bigwedge M[[t]])$, define a product $D_{t} * D_{t}^{\prime}$ through the equality: $\left(D_{t} * D_{t}^{\prime}\right) \alpha=\jmath\left(D_{t}\right)\left(D_{t}^{\prime} \alpha\right)$. Clearly $\jmath\left(D_{t}\right) \alpha=D_{t} \alpha$ for each $\alpha \in \bigwedge M$ and

$$
\begin{aligned}
\left(D_{t} * D_{t}^{\prime}\right)(\alpha) & =\sum_{h \geq 0}\left(\sum_{i+j=h} D_{i}\left(D_{j}^{\prime} \alpha\right)\right) t^{h}=\jmath\left(D_{t}\right)\left(\sum_{j \geq 0} D_{j}^{\prime} \alpha \cdot t^{j}\right)= \\
& =\jmath\left(D_{t}\right)\left(D_{t}^{\prime} \alpha\right)=\left(\jmath\left(D_{t}\right) \circ \jmath\left(D_{t}^{\prime}\right)\right) \alpha .
\end{aligned}
$$

2.5 The product $D_{t} * D_{t}^{\prime}$ of two derivations on $\bigwedge M$ is a derivation on $\bigwedge M$. Indeed, using (3) and (4):

$$
\begin{aligned}
\left(D_{t} * D_{t}^{\prime}\right)(\alpha \wedge \beta) & =\jmath\left(D_{t}\right)\left(D_{t}^{\prime}(\alpha \wedge \beta)\right)=\jmath\left(D_{t}\right)\left(D_{t}^{\prime} \alpha \wedge D_{t}^{\prime} \beta\right)= \\
& =\jmath\left(D_{t}\right)\left(D_{t}^{\prime} \alpha\right) \wedge \jmath\left(D_{t}\right)\left(D_{t}^{\prime} \beta\right)=\left(D_{t} * D_{t}^{\prime}\right) \alpha \wedge\left(D_{t} * D_{t}^{\prime}\right) \beta,
\end{aligned}
$$

as desired. Let now $D^{(1)}=\left(D_{i}^{(1)}\right)_{i \geq 0}$ be any (possibly finite) sequence of endomorphisms of $M$ and, for each $m \in M$, let $D_{t}^{(1)}(m)=\sum_{i \geq 0} D_{i}^{(1)}(m) t^{i}$. Then $D_{t}^{(1)}: M \rightarrow$ $M[[t]]$ is an $A$-module homomorphism.

2.6 Proposition. There exists a unique derivation $D_{t}: \bigwedge M \rightarrow \bigwedge M[[t]]$ such that $D_{\left.t\right|_{M}}=D_{t}^{(1)}$ (or, equivalently, $D_{\left.i\right|_{M}}=D_{i}^{(1)}$ ).

Proof. For each $k \geq 1$, consider the $A$-multilinear map $M^{\otimes k} \rightarrow\left(\bigwedge^{k} M\right)[[t]]$ defined by $m_{i_{1}} \otimes \ldots \otimes m_{i_{k}} \mapsto D_{t}^{(1)} m_{i_{1}} \wedge \ldots \wedge D_{t}^{(1)} m_{i_{k}}$, which is clearly alternating. By the universal property of exterior powers, it factors through a unique $A$-module homomorphism $\bigwedge^{k} M \rightarrow\left(\bigwedge^{k} M\right)[[t]]$, given by $D_{t}^{(k)}\left(m_{i_{1}} \wedge \ldots \wedge m_{i_{k}}\right)=D_{t}^{(1)} m_{i_{1}} \wedge \ldots \wedge D_{t}^{(1)} m_{i_{k}}$ on monomials. Let $D_{t} \alpha=D_{t}^{(k)} \alpha$ for all $\alpha \in \Lambda^{k} M$ and all $k \geq 0$. It follows that if $\alpha \in \bigwedge^{k_{1}} M$ and $\beta \in \bigwedge^{k_{2}} M$, equation (2) holds by definition of $D_{t}$ and the fact that $\alpha \wedge \beta$ is a finite $A$-linear combination of elements of the form

$$
\left\{m_{i_{1}} \wedge \ldots \wedge m_{i_{k_{1}}} \wedge m_{i_{k_{1}+1}} \wedge \ldots \wedge m_{i_{k_{1}+k_{2}}} ; \quad 1 \leq i_{1}<\ldots<i_{k_{1}+k_{2}}\right\}
$$


Since any element of $\bigwedge M$ is a finite sum of homogeneous ones, equation (2D) holds for any arbitrary pair as well. The unicity part is straightforward: were $D_{t}^{\prime}$ another extension of $D_{t}^{(1)}$, one would have $D_{t}^{\prime}\left(m_{i_{1}} \wedge \ldots \wedge m_{i_{k}}\right)=D_{t}^{(1)} m_{i_{1}} \wedge \ldots \wedge D_{t}^{(1)} m_{i_{k}}=$ $D_{t}\left(m_{i_{1}} \wedge \ldots \wedge m_{i_{k}}\right)$, for each $m_{i_{1}} \wedge \ldots \wedge m_{i_{k}}$ and each $k \geq 1$. Hence $D_{t}^{\prime}=D_{t}$.

2.7 Let $\mathcal{S}_{t}(\bigwedge M)$ be the set of all derivations $D_{t}:=\sum_{i>0} D_{i} t^{i}$ such that $D_{i_{\mid}} \in$ $\operatorname{End}_{A}(M)$ (i.e. the submodule $M$ of $\bigwedge M$ is $D_{i}$-stable) and $D_{\left.0\right|_{M}}$ is an isomorphism. Hence $D_{0}: \bigwedge M \rightarrow \bigwedge M$ is an isomorphism too.

2.8 Proposition. The pair $\left(\mathcal{S}_{t}(\bigwedge M), *\right)$ is a group.

Proof. By 2.5, $\mathcal{S}_{t}(\bigwedge M)$ is closed under $*$. By its very definition, $*$ is associative. The map $1: \bigwedge M \rightarrow(\bigwedge M)[[t]]$, sending any $\alpha \in \bigwedge M$ to itself, thought of as a constant formal power series, is the $*$-neutral element. Thinking to $D_{t}$ as a formal power series with coefficients in $\operatorname{End}_{A}(\bigwedge M)$, the formal inverse $D_{t}^{-1}$ of $D_{t}$ (existing because of the invertibility of $\left.D_{0}\right)$ is a derivation as well. In fact

$$
\begin{aligned}
D_{t}^{-1}(\alpha \wedge \beta) & =\jmath\left(D_{t}^{-1}\right)\left(\left(D_{t} * D_{t}^{-1}\right) \alpha \wedge\left(D_{t} * D_{t}^{-1}\right) \beta\right)= \\
& =\jmath\left(D_{t}^{-1}\right)\left(\jmath\left(D_{t}\right) D_{t}^{-1} \alpha \wedge \jmath\left(D_{t}\right) D_{t}^{-1} \beta\right)= \\
& =\left(\jmath\left(D_{t}^{-1}\right) \circ \jmath\left(D_{t}\right)\right)\left(D_{t}^{-1} \alpha \wedge D_{t}^{-1} \beta\right)=D_{t}^{-1} \alpha \wedge D_{t}^{-1} \beta,
\end{aligned}
$$

since $D_{t}^{-1} * D_{t}=D_{t} * D_{t}^{-1}=\mathbf{1}$.

2.9 We fix another piece of notation. Let $A[\mathbf{T}]$ be the polynomial ring in infinitely many indeterminates $\mathbf{T}=\left(T_{1}, T_{2}, \ldots\right)$. For each $k$-tuple $I:=\left(i_{1}, \ldots, i_{k}\right)$ of positive integers, we denote by $\Delta_{I}(\mathbf{T}):=\Delta_{\left(i_{1}, \ldots, i_{k}\right)}(\mathbf{T})$ the Schur polynomial $\operatorname{det}\left[\left(T_{i_{j}-i}\right)_{1 \leq i, j \leq k}\right] \in$ $A[\mathbf{T}]$ (setting $T_{0}=1$ and $T_{j}=0$, if $j<0$ ). By expanding $\Delta_{I}(\mathbf{T})$ along the last column, one sees that $\Delta_{I}(\mathbf{T})$ belongs to the ideal $\left(T_{i_{k}-1}, \ldots, T_{i_{k}-k}\right)$ of $A[\mathbf{T}]$. In particular $\Delta_{(2,3, \ldots, h+1)}(\mathbf{T}) \in\left(T_{1}, \ldots, T_{h}\right)$. If $D:=\left(D_{0}, D_{1}, \ldots,\right)$ is the sequence of coefficients of some $D_{t} \in \mathcal{S}_{t}(\bigwedge M)$ such that $D_{0}=i d_{\wedge M}$, one defines $\Delta_{I}(D)$ to be the evaluation of $\Delta_{I}(\mathbf{T})$ at $D$ (via the substitution $T_{i} \mapsto D_{i}$ ).

2.10 For each $i \geq 0$, define $\bar{D}_{i} \in \operatorname{End}_{A}(\bigwedge M)$ via the equality $D_{t}^{-1}=\sum_{i>0}(-1)^{i} \bar{D}_{i} t^{i}$. By equating the coefficients of the same power of $t$ on both sides of the equation $D_{t} * D_{t}^{-1}=1$, one gets $\bar{D}_{0}=D_{0}^{-1}$, while, for each $h \geq 1$ :

$$
\bar{D}_{h}-\bar{D}_{h-1} D_{1}+\ldots+(-1)^{h} D_{h}=0,
$$

so that, e.g., $\bar{D}_{1}=D_{1}, \bar{D}_{2}=D_{1}^{2}-D_{2}$. In general, one has (see [2], Appendix A):

$$
\bar{D}_{h}=\Delta_{(2,3, \ldots, h+1)}(D) \text {. }
$$

2.11 Proposition (Integration by parts). Let $D_{t} \in \mathcal{S}_{t}(\bigwedge M)$. Then:

$D_{h} \alpha \wedge \beta=\sum_{i \geq 0}(-1)^{i} D_{h-i}\left(\alpha \wedge \bar{D}_{i} \beta\right)=D_{h} \alpha \wedge \beta-D_{h-1} \alpha \wedge \bar{D}_{1} \beta+\ldots+(-1)^{i} D_{0} \alpha \wedge \bar{D}_{h} \beta$.

Proof. One expands both sides of the equality $\jmath\left(D_{t}\right)\left(\alpha \wedge D_{t}^{-1} \beta\right)=D_{t} \alpha \wedge \beta$, and then compares the coefficients of $t^{h}$ occurring on each side.

2.12 Example. One has $D_{1} \alpha \wedge D_{0} \beta=D_{1}(\alpha \wedge \beta)-D_{0} \alpha \wedge \bar{D}_{1} \beta$ and:

$$
D_{2} \alpha \wedge D_{0} \beta=D_{2}(\alpha \wedge \beta)-D_{1}\left(\alpha \wedge \bar{D}_{1} \beta\right)+D_{0} \alpha \wedge \bar{D}_{2} \beta \text {. }
$$




\section{Schubert Calculus on a Grassmann Algebra}

3.1 From now on, $A$ will be assumed to be any graded ring $\bigoplus_{i>0} A_{i}$ such that $A_{0}=\mathbb{Z}$. Let $X$ be an indeterminate over $A, M:=X A[X]$ and $M(\mathrm{p}):=M / \mathrm{p} M$, where $\mathrm{p}$ is either the 0 polynomial or a monic polynomial $X^{n}-e_{1} X^{n-1}+\ldots+(-1)^{n} e_{n} \in A[X]$ such that $e_{i} \in A_{i}$. Then $M(\mathrm{p})$ is a free $A$-module generated by $\boldsymbol{\epsilon}=\left(\epsilon^{i}\right)_{1 \leq i \leq n}$, where $n$ is either $\operatorname{deg}(\mathrm{p})$ if $\mathrm{p} \neq 0$, or $\infty$ if $\mathrm{p}=0$.

3.2 Let $\mathcal{I}^{k}=\left\{I=\left(i_{1}, \ldots, i_{k}\right) \in \mathbb{N}^{k} \mid 1 \leq i_{1}<\ldots<i_{k}\right\}$ (as in [3], §5, Section 1, and [4]). The weight of $I \in \mathcal{I}^{k}$ is $w t(I)=\sum_{j=1}^{k}\left(i_{j}-j\right)$. It coincides with the weight of the associated partition $\left(i_{k}-k, i_{k-1}-(k-1), \ldots, i_{1}-1\right)$. If $I:=\left(i_{1}, \ldots, i_{k}\right) \in \mathcal{I}^{k}$, let $\wedge^{I} \boldsymbol{\epsilon}$ denote $\epsilon^{i_{1}} \wedge \ldots \wedge \epsilon^{i_{k}}$. Each exterior power $\wedge^{k} M(\mathrm{p})$ is a free $A$-module with basis $\wedge^{k} \epsilon:=\left\{\wedge^{I} \epsilon: I \in \mathcal{I}_{n}^{k}\right\}$. If $a \in A_{h}$, the weight of $a \cdot \wedge^{I} \boldsymbol{\epsilon}$ is, by definition, $h+w t(I)$. Set $\left(\bigwedge^{k} M(\mathrm{p})\right)_{w}=\bigoplus_{0 \leq h \leq w}\left(\bigoplus_{w t(I)=h} A_{w-h} \cdot \wedge^{I} \boldsymbol{\epsilon}\right)$. Then $\bigwedge^{k} M(\mathrm{p})=\bigoplus_{w \geq 0}\left(\bigwedge^{k} M(\mathrm{p})\right)_{w}$, a graded $A$-module via weight.

3.3 By Proposition 2.6 there is a unique sequence $D:=\left(D_{0}, D_{1}, \ldots\right)$ of $A$-endomorphisms of $\bigwedge M(\mathrm{p})$ such that i) (the $h^{\text {th }}$-order) Leibniz's rule (1) holds for each $h \geq 0$ and each $\alpha, \beta \in \bigwedge^{k} M(\mathrm{p})$ and ii) the initial conditions $D_{h} \epsilon^{i}=\epsilon^{i+h}$ are satisfied, for each $h \geq 0$ and each $i \geq 1$. Notice that $D_{i} \circ D_{j}=D_{j} \circ D_{i}$ in $\operatorname{End}_{A}(\bigwedge M(\mathrm{p}))$, as a simple induction shows.

3.4 Proposition. The following formula holds:

$$
D_{h} \epsilon^{i_{1}} \wedge \ldots \wedge \epsilon^{i_{k}}=\sum \epsilon^{i_{1}+h_{1}} \wedge \ldots \wedge \epsilon^{i_{k}+h_{k}},
$$

the sum over all $h$-tuples $\left(h_{i}\right)_{1 \leq i \leq k}$ of non negative integers such that $h_{1}+\ldots+h_{k}=h$.

Proof. See [8] or, since equality (8) is defined over the integers, use the same inductive proof as in [4], Proposition 2.3.

3.5 Example. When expanding $D_{h} \epsilon^{i_{1}} \wedge \ldots \wedge \epsilon^{i_{k}}$, cancellations may occur on the right hand side of (8), due to the $\mathbb{Z}_{2}$-symmetry of the $\wedge$-product. For instance:

$$
D_{2}\left(\epsilon^{1} \wedge \epsilon^{2}\right)=\epsilon^{3} \wedge \epsilon^{2}+\epsilon^{2} \wedge \epsilon^{3}+\epsilon^{1} \wedge \epsilon^{4}=\epsilon^{1} \wedge \epsilon^{4} .
$$

The surviving summands are predicted by Pieri's formula for $D_{h}$, a rule to speed up computations of "derivatives" of $k$-vectors.

3.6 Theorem (Pieri's formula). Pieri's formula holds:

$$
D_{h}\left(\epsilon^{i_{1}} \wedge \ldots \wedge \epsilon^{i_{k}}\right)=\sum_{\left(h_{i}\right) \in P(I, h)} \epsilon^{i_{1}+h_{1}} \wedge \ldots \wedge \epsilon^{i_{k}+h_{k}},
$$

where, if $I=\left(i_{1}, \ldots, i_{k}\right) \in \mathcal{I}^{k}$, we denote by $\mathcal{P}(I, h)$ the set of all $k$-tuples of non negative integers $\left(h_{1}, \ldots, h_{k}\right)$ such that $i_{1}+h_{1}<i_{2} \leq i_{2}+h_{2}<\ldots<i_{k-1} \leq i_{k}$ and $h_{1}+\ldots+h_{k}=h$.

Proof. See [8] or, since formula (9) is defined over the integers, use the same proof as in 4], Theorem 2.4 . 
3.7 Let $A$ be as in 3.1 and $A[\mathbf{T}]$ be as in 2.10. If $a \in A_{l}$, the degree of the monomial $a T_{i_{1}}^{m_{1}} \ldots T_{i_{j}}^{m_{j}}$ is defined to be $l+m_{1} i_{1}+\ldots+m_{j} i_{j}$. Then $A[\mathbf{T}]$ is itself a graded ring $\bigoplus_{h \geq 0} A[\mathbf{T}]_{h}$, where $A[\mathbf{T}]_{h}$ is the submodule of all elements of $A[\mathbf{T}]$ of degree $h$. There is a natural evaluation map, $\mathrm{ev}_{D}: A[\mathbf{T}] \rightarrow \operatorname{End}_{A}(\bigwedge M(\mathrm{p}))$, sending $P \in A[\mathbf{T}]$ to $P(D)$ (got by "substituting" $T_{i} \mapsto D_{i}$ into $\left.P\right)$. We denote by $\mathcal{A}^{*}(\bigwedge M(\mathrm{p}))$ the image of $\operatorname{ev}_{D}$ in $\operatorname{End}_{A}(\bigwedge M(\mathrm{p}))$ and by $\mathcal{A}^{*}\left(\bigwedge^{k} M(\mathrm{p})\right)$ the image of the natural restriction map

$$
\rho_{k}: \mathcal{A}^{*}(\bigwedge M(\mathrm{p})) \rightarrow \operatorname{End}_{A}\left(\bigwedge^{k} M(\mathrm{p})\right),
$$

given by $P(D) \mapsto(D)_{\left.\right|_{\Lambda^{k} M(\mathrm{p})}}$. Pieri's formula implies Giambelli's formula, a special case of the general determinantal formula stated in [8], Main Theorem, which reads, in this case, as:

$$
\epsilon^{i_{1}} \wedge \ldots \wedge \epsilon^{i_{k}}=\Delta_{\left(i_{1} \ldots i_{k}\right)}(D) \cdot \epsilon^{1} \wedge \ldots \wedge \epsilon^{k}
$$

where, as in 2.10 $\Delta_{\left(i_{1} \ldots i_{k}\right)}(D)=\operatorname{ev}_{D}\left(\Delta_{\left(i_{1} \ldots i_{k}\right)}(\mathbf{T})\right)$. We have hence shown that:

3.8 Theorem. The natural evaluation map $\operatorname{ev}_{\epsilon^{1} \wedge \ldots \wedge \epsilon^{k}}: \mathcal{A}^{*}(\bigwedge M(\mathrm{p})) \rightarrow \bigwedge^{k} M(\mathrm{p})$, mapping $P(D) \mapsto P(D) \epsilon^{1} \wedge \ldots \wedge \epsilon^{k}$ is surjective.

3.9 It follows that $\operatorname{ker}\left(\rho_{k}\right)=\operatorname{ker}\left(\operatorname{ev}_{\epsilon^{1} \wedge \ldots \wedge \epsilon^{k}}\right)$ and then:

$$
\mathcal{A}^{*}\left(\bigwedge^{k} M(\mathrm{p})\right)=\frac{\mathcal{A}^{*}(\bigwedge M(\mathrm{p}))}{\operatorname{ker}\left(\mathrm{ev}_{\epsilon^{1} \wedge \ldots \wedge \epsilon^{k}}\right)} .
$$

The induced map $\Pi_{k}: \mathcal{A}^{*}\left(\bigwedge^{k} M(\mathrm{p})\right) \rightarrow \bigwedge^{k} M(\mathrm{p})$, defined by

$$
P(D)+\operatorname{ker}_{\epsilon^{1} \wedge \ldots \wedge \epsilon^{k}} \mapsto P(D) \epsilon^{1} \wedge \ldots \wedge \epsilon^{k},
$$

we call the Poincaré isomorphism.

3.10 Remark. Let $\mathcal{I}_{n}^{k}=\left\{I \in \mathcal{I}^{k} \mid i_{k} \leq n\right\}$. A routine check shows that if $I=$ $\left(i_{1}, \ldots, i_{k}\right) \in \mathcal{I}_{n}^{k}$ and $H \in \mathcal{P}(I, h)$ then $I+H:=\left(i_{1}+h_{1}, \ldots, i_{k}+h_{k}\right) \in \mathcal{I}^{k}$. Denote by $\mathcal{I}^{k, w}$ the set of all $I \in \mathcal{I}^{k}$ such that $w t(I)=w$. Combining Pieri's formula (9) with Giambelli's formula (10), one has, for each $I \in \mathcal{I}^{k}$ and each $h \geq 0$ :

$D_{h} \Delta_{I}(D) \epsilon^{1} \wedge \ldots \wedge \epsilon^{k}=D_{h} \cdot \wedge^{I} \boldsymbol{\epsilon}=\sum_{H \in \mathcal{P}(I, h)} \wedge^{I+H} \boldsymbol{\epsilon}=\sum_{H \in \mathcal{P}(I, h)} \Delta_{I+H}(D) \epsilon^{1} \wedge \ldots \wedge \epsilon^{k}$,

proving the equality $D_{h} \Delta_{I}(D)=\sum_{H \in \mathcal{P}(I, h)} \Delta_{I+H}(D)$ in the $\operatorname{ring} \mathcal{A}^{*}\left(\bigwedge^{k} M(\mathrm{p})\right)$.

3.11 Remark (see 9]). Let $\operatorname{Split}_{A}^{k}(\mathrm{p})$ be the universal splitting algebra of the monic polynomial $\mathrm{p}$ into the product of two monic polynomials, one of degree $k$. Let $\mathrm{p}=\mathrm{p}_{1} \mathrm{q}$ be the universal splitting of $\mathrm{p}$ in $\operatorname{Split}_{A}^{k}(\mathrm{p})$, where $\operatorname{deg}\left(\mathrm{p}_{1}\right)=k$, and denote by $s_{i}$ the complete symmetric polynomial of degree $i$ in the universal roots of $\mathrm{p}_{1}$. Then $\operatorname{Split}_{A}^{k}(\mathrm{p})$ is generated, as an $A$-algebra, by $\left(s_{i}\right)_{i \geq 1}$ and the map $\mathcal{A}^{*}\left(\bigwedge^{k} M(\mathrm{p})\right) \rightarrow S p l i t_{A}^{k}(\mathrm{p})$, defined by $D_{i} \mapsto s_{i}$, is an $A$-algebra isomorphism. This is because of the module structure of $\bigwedge^{k} A[X]$ over the ring of symmetric functions defined and studied in $[8$. In fact our 
formula (9) is the same as Pieri's formula (2.1.1) of [8], after replacing $s_{i}$ with $D_{i}$. Let $p: E \rightarrow \mathcal{Y}$ be a vector bundle of rank $n$ and let $p_{k}: G(k, E) \rightarrow \mathcal{Y}$ be the Grassmann bundle over $\mathcal{Y}$ of $k$-planes in the fibers of $E$. In [9] the authors show that, if $A:=A^{*}(\mathcal{Y})$ is the Chow ring of $\mathcal{Y}$ and $\mathrm{p}=X^{n}+c_{1} X^{n-1}+\ldots+c_{n} \in A[X]$ is such that $c_{i}:=c_{i}(E)$ are the Chern classes of $E$, there is an isomorphism $\operatorname{Split}_{A}^{k}(\mathrm{p}) \rightarrow A^{*}(G(k, E))$. Let $\mathcal{Q}_{k}$ be the universal quotient bundle over $G(k, E)$. Then, the same proof as in 9 ] works using derivations: by the basis theorem ([2, p. 268) the unique $A$-module homomorphism $\iota_{k}: A^{*}(G(k, E)) \rightarrow \mathcal{A}^{*}\left(\bigwedge^{k} M(\mathrm{p})\right)$, mapping $\Delta_{I}\left(c\left(\mathcal{Q}_{k}-p_{k}^{*} E\right)\right.$ to $\Delta_{I}(D)$, is certainly an isomorphism. To check that it is also a ring homomorphism, it is sufficient to check it on products of the form $c_{h}\left(\mathcal{Q}_{k}-p_{k}^{*} E\right) \cdot \Delta_{I}\left(c\left(\mathcal{Q}_{k}-p_{k}^{*} E\right)\right.$ :

$$
\begin{aligned}
\iota_{k}\left(c_{h}\left(\mathcal{Q}_{k}-p_{k}^{*} E\right) \cdot \Delta_{I}\left(c\left(\mathcal{Q}_{k}-p_{k}^{*} E\right)\right)\right. & =\iota_{k}\left(\sum_{H \in \mathcal{P}(I, h)} \Delta_{I+H}\left(c\left(\mathcal{Q}_{k}-p_{k}^{*} E\right)\right)\right)= \\
=\sum_{H \in \mathcal{P}(I, h)} \Delta_{I+H}(D)=D_{h} \Delta_{I}(D) & =\iota_{k}\left(c _ { h } ( \mathcal { Q } _ { k } - p _ { k } ^ { * } E ) \cdot \iota _ { k } \left(\Delta _ { I } \left(\left(c\left(\mathcal{Q}_{k}-p_{k}^{*} E\right)\right),\right.\right.\right.
\end{aligned}
$$

by [2, Proposition 14.6.1, and 3.10

3.12 Remark. Theorem 3.8 can be proven by showing that for each $I \in \mathcal{I}^{k}$, there exists $G_{I} \in A[\mathbf{T}]$ such that $\wedge^{I} \boldsymbol{\epsilon}=G_{I}(D) \cdot \epsilon^{1} \wedge \ldots \wedge \epsilon^{k}$. This can be achieved via integration by parts (7), as follows. We say that $\bigwedge^{k} M(\mathrm{p})$ enjoys the property $\mathbf{G}_{j}$, for some $1 \leq j \leq k$, if, for each $i_{j+1}<\ldots<i_{k}$ such that $j<i_{j+1}$, there exists a polynomial $G_{j, i_{j+1}, \ldots, i_{k}} \in A[\mathbf{T}]$ such that $\epsilon^{1} \wedge \ldots \wedge \epsilon^{j} \wedge \epsilon^{i_{j+1}} \wedge \ldots \wedge \epsilon^{i_{k}}=G_{j, i_{j+1}, \ldots, i_{k}}(D) \cdot \epsilon^{1} \wedge \ldots \wedge \epsilon^{k}$. We shall show, by descending induction, that $\bigwedge^{k} M(\mathrm{p})$ enjoys $\mathbf{G}_{j}$ for each $1 \leq j \leq k$. In fact $\mathbf{G}_{k}$ is trivially true.

Let us suppose that $\mathbf{G}_{j}$ holds for some $2 \leq j \leq k-1$. Then $\mathbf{G}_{j-1}$ holds. In fact, for each $j-1<i_{j}<\ldots<i_{k}$,

$$
\epsilon^{1} \wedge \ldots \wedge \epsilon^{j-1} \wedge \epsilon^{i_{j}} \wedge \ldots \wedge \epsilon^{i_{k}}=D_{i_{j}-j}\left(\epsilon^{1} \wedge \ldots \wedge \epsilon^{j-1} \wedge \epsilon^{j}\right) \wedge \epsilon^{i_{j+1}} \wedge \ldots \wedge \epsilon^{i_{k}},
$$

basically by [4, Corollary 2.5. By applying integration by parts (7), one gets:

$$
\epsilon^{1} \wedge \ldots \wedge \epsilon^{j-1} \wedge \epsilon^{i_{j}} \wedge \ldots \wedge \epsilon^{i_{k}}=\sum_{h=0}^{i_{j}-j} D_{i_{j}-j-h}\left(\epsilon^{1} \wedge \ldots \wedge \epsilon^{j} \wedge \bar{D}_{h}\left(\epsilon^{i_{j+1}} \wedge \ldots \wedge \epsilon^{i_{k}}\right)\right) .
$$

But $\bar{D}_{h}\left(\epsilon^{i_{j+1}} \wedge \ldots \wedge \epsilon^{i_{k}}\right)$ is a sum of elements of the form $\epsilon^{h_{j+1}} \wedge \ldots \wedge \epsilon^{h_{k}}$, with $j<h_{j+1}<\ldots<h_{j}$. Then, by the inductive hypothesis, one concludes that $\mathbf{G}_{j-1}$ holds, too. In particular $\mathbf{G}_{1}$ holds and the claim is proven.

\section{Presentations for Intersection Rings}

4.1 Proposition. Let $D_{t}^{-1}:=\sum_{j \geq 0}(-1)^{j} \bar{D}_{j} t^{j}$ be the inverse of $D_{t} \in \mathcal{S}_{t}(\bigwedge M(\mathrm{p}))$. Then $\bar{D}_{\left.h\right|_{\wedge^{k} M(\mathrm{p})}}=0$, for each $h>k$. 
Proof. By induction on $k$. If $k=0$ one has $\bar{D}_{h}(m)=0$, for each $h \geq 2$ and each $m \in M(\mathrm{p})$. In fact, if $m \in M(\mathrm{p}), D_{t} m=\sum_{i \geq 0} D_{1}^{i} m \cdot t^{i}$. Therefore $D_{t}^{-1} m=1-D_{1} m \cdot t$, i.e. $\bar{D}_{h_{\mid M(\mathrm{p})}}=0$ for each $h \geq 2$. Suppose now the property true for $k-1$ and let $h>k$. Any $m_{k} \in \Lambda^{k} M(\mathrm{p})$ is a finite $A$-linear combination of elements of the form $m \wedge m_{k-1}$, for suitable $m \in M(\mathrm{p})$ and $m_{k-1} \in \bigwedge^{k-1} M(\mathrm{p})$. It suffices then to check the property for elements of this form. One has: $\bar{D}_{h}\left(m \wedge m_{k-1}\right)=\sum_{j=0}^{h} \bar{D}_{j} m \wedge \bar{D}_{h-j}\left(m_{k-1}\right)$. As $\bar{D}_{j} m=0$, for $j \geq 2$, it follows that $\sum_{j=0}^{h} \bar{D}_{j} m \wedge \bar{D}_{h-j} m_{k-1}=\bar{D}_{1} m \wedge \bar{D}_{h-1} m_{k-1}$. By the inductive hypothesis, this last term vanishes as well, because $h-1>k-1$.

In the sequel $M$ will be as in 3.1 (i.e. $M(\mathrm{p})$ for $\mathrm{p}=0$ ).

4.2 Proposition. The ring $\mathcal{A}^{*}\left(\bigwedge^{k} M\right)$ is generated by $\left(D_{1}, D_{2}, \ldots, D_{k}\right)$ as an $A$ algebra.

Proof. Let $D_{t}^{-1}=\sum_{i>0}(-1)^{i} \bar{D}_{i} t^{i}$ be the inverse of $D_{t}$. First one observes that, for each $h \geq 1, \bar{D}_{h}=\operatorname{ev}_{D}\left(\Delta_{(2,3, \ldots, h+1)}(\mathbf{T})\right)=\Delta_{(2,3, \ldots, h+1)}(D)$ and that $\Delta_{(2,3, \ldots, h+1)}(\mathbf{T}) \in$ $A[\mathbf{T}]$ lands in fact in the subring $A\left[T_{1}, \ldots, T_{h}\right]$ of $A[\mathbf{T}]$, by Remark 2.9, One knows that $\bar{D}_{k+j}=0$ in $\mathcal{A}^{*}\left(\bigwedge^{k} M(\mathrm{p})\right)$ for each $j \geq 1$ (Proposition 4.1). Working modulo $\operatorname{ker}\left(\rho_{k}\right)$ (see 3.7) we may hence write:

$$
\sum_{i \geq 0} D_{i} t^{i}=\frac{1}{1-\bar{D}_{1} t+\bar{D}_{2} t^{2}+\ldots+(-1)^{k} \bar{D}_{k} t^{k}} .
$$

Define $\widetilde{D}_{j}\left(\mathbf{T}_{k}\right) \in A\left[T_{1}, \ldots, T_{k}\right] \subseteq A[\mathbf{T}]$ as

$$
\sum_{j \geq 0} \widetilde{D}_{j}\left(\mathbf{T}_{k}\right) t^{i}=\frac{1}{1-\Delta_{(2)}(\mathbf{T}) t+\Delta_{(23)}(\mathbf{T}) t^{2}+\ldots+(-1)^{k} \Delta_{(23 \ldots k+1)}(\mathbf{T}) t^{k}} .
$$

One clearly has that $D_{j}-\widetilde{D}_{j}\left(\mathbf{D}_{k}\right) \in \operatorname{ker}\left(\rho_{k}\right)$ for each $j \geq 0$. Moreover, if $1 \leq j \leq k$, $\widetilde{D}_{j}\left(\mathbf{T}_{k}\right)=T_{j}$, proving the claim.

4.3 Example. In $\mathcal{A}^{*}\left(\bigwedge^{2} M\right)$ one has, using the recipe (12):

$$
\widetilde{D}_{3}\left(\mathbf{T}_{2}\right):=\widetilde{D}_{3}\left(T_{1}, T_{2}\right)=T_{2} T_{1}-T_{1}\left(T_{1}^{2}-T_{2}\right)=-T_{1}^{3}+2 T_{1} T_{2}
$$

and

$\widetilde{D}_{4}\left(\mathbf{T}_{2}\right)=\widetilde{D}_{3} T_{1}-T_{2}\left(T_{1}^{2}-T_{2}\right)=\left(-T_{1}^{3}+2 T_{1} T_{2}\right) T_{1}-T_{2}\left(T_{1}^{2}-T_{2}\right)=-T_{1}^{4}+T_{1}^{2} T_{2}+T_{2}^{2}$.

4.4 Proposition. Let $P \in A\left[T_{1}, \ldots, T_{k}\right]_{w} \subset A[\mathbf{T}]_{w}$ such that $P(D) \epsilon^{1} \wedge \ldots \wedge \epsilon^{k}=0$ $(w \geq 0)$. Then $P=0$.

Proof. Any polynomial $P \in A\left[T_{1}, \ldots, T_{k}\right]$ of degree $w$ is a unique $A$-linear combination of $\Delta_{I}(\mathbf{T})$, with $I \in \mathcal{I}^{k, w}$ (since the Schur polynomials $\left\{\Delta_{I}(\mathbf{T}) \mid I \in \mathcal{I}^{k}\right\}$ are a $\mathbb{Z}$-basis of $\mathbb{Z}[\mathbf{T}]$ ). Hence $P=\sum_{I \in \mathcal{I}^{k, w}} a_{I} \Delta_{I}(\mathbf{T})$ for some (unique!) $a_{I} \in A_{w-w t(I)}$ and if $P(D) \epsilon^{1} \wedge \ldots \wedge \epsilon^{k}=0$, then:

$$
0=P(D) \cdot \epsilon^{1} \wedge \ldots \wedge \epsilon^{k}=\sum_{I \in \mathcal{I}^{k, w}} a_{I} \Delta_{I}(D) \cdot \epsilon^{1} \wedge \ldots \wedge \epsilon^{k}=\sum_{I \in \mathcal{I}^{k, w}} a_{I} \cdot \wedge^{I} \boldsymbol{\epsilon} .
$$


Since $\left\{\wedge^{I} \boldsymbol{\epsilon}\right\}_{I \in I^{k, w}}$ are $A$-linearly independent, $a_{I}=0$ for all $I \in \mathcal{I}^{k, w}$, i.e. $P=0$.

4.5 Corollary. The map $3.7, \mathrm{ev}_{D}: A[\mathbf{T}] \longrightarrow \mathcal{A}^{*}(\bigwedge M)$ is an isomorphism. Hence:

$$
\mathcal{A}^{*}(\bigwedge M)=A[D]:=A\left[D_{1}, D_{2}, \ldots\right] \cong A[\mathbf{T}],
$$

the polynomial ring in infinitely many indeterminates, while

$$
\mathcal{A}^{*}\left(\bigwedge^{k} M\right)=A\left[\mathbf{D}_{k}\right]:=A\left[D_{1}, D_{2}, \ldots, D_{k}\right]
$$

Proof. Apply Proposition 4.4 One may assume that $P \in A[\mathbf{T}]$ is homogeneous of degree $w \geq 0$. Suppose that $\operatorname{ev}_{D}(P)=P(D)=0 \in \mathcal{A}^{*}(\bigwedge M)$. There is $k \geq 1$ such that $P \in A\left[T_{1}, T_{2}, \ldots, T_{k}\right]$. But then $P(D) \cdot \epsilon^{1} \wedge \ldots \wedge \epsilon^{k}=0$ implies $P=0$, because otherwise one would have a relation (of degree $w$ ), whence (15). Since $\mathcal{A}^{*}\left(\bigwedge^{k} M\right)=$ $\rho_{k}\left(\mathcal{A}^{*}(\bigwedge M)\right)$ and, by Proposition 4.1. $\rho_{k}\left(\bar{D}_{h}\right)=0$ for all $h \geq k+1$, one gets the presentation (16).

4.6 For each $i \geq 1$, let $\nu^{q n+i}=(\mathrm{p}(X))^{q} X^{i}$. Then $\boldsymbol{\nu}=\left(\nu^{1}, \nu^{2}, \ldots\right)$ is an $A$-basis of $M:=M(0)$, such that $\nu^{i}=X^{i}$ for each $1 \leq i \leq n$. Let $\wedge M \wedge \mathrm{p} M:=\bigoplus_{k \geq 1} \wedge^{k-1} M \wedge$ $\mathrm{p} M$ be the bilateral ideal of $\bigwedge M$ generated by p. As $\mathrm{p} M$ is the submodule of $M$ generated by $\nu^{i}$ with $i>n$, the submodule $\wedge^{k-1} M \wedge \mathrm{p} M$ is the $A$-submodule of $\wedge^{k} M$ generated by $\nu^{i_{1}} \wedge \ldots \wedge \nu^{i_{k}}$, with $i_{k}>n$. The natural map $\wedge M \rightarrow \wedge M(\mathrm{p}) \quad$ (resp. $\left.\bigwedge^{k} M \rightarrow \bigwedge^{k} M(\mathrm{p})\right)$ is surjective and has kernel $\wedge M \wedge \mathrm{p} M\left(\right.$ resp. $\left.\bigwedge^{k-1} M \wedge \mathrm{p} M\right)$. Hence, one has canonical isomorphisms

$$
\bigwedge M(\mathrm{p})=\frac{\bigwedge M}{\bigwedge M \wedge \mathrm{p} M} \quad \text { and } \quad \bigwedge^{k} M(\mathrm{p})=\frac{\bigwedge^{k} M}{\bigwedge^{k-1} M \wedge \mathrm{p} M}
$$

Let $\phi_{k}: \bigwedge^{k} M \rightarrow \bigwedge^{k} M(\mathrm{p})$ be the canonical projection and let

$$
J_{k}(\mathrm{p}):=\left\{P(D) \in A\left[D_{1}, \ldots, D_{k}\right] \mid P(D) \epsilon^{1} \wedge \ldots \wedge \epsilon^{k} \in \bigwedge^{k-1} M \wedge \mathrm{p} M\right\},
$$

which is an ideal of $A\left[D_{1}, \ldots, D_{k}\right]=\mathcal{A}^{*}\left(\bigwedge^{k} M\right)$.

4.7 Theorem. For each $j \geq 1$, let

$$
\widetilde{D}_{n-k+j}\left(\mathbf{D}_{k}, \mathbf{p}\right)=\widetilde{D}_{n-k+j}\left(\mathbf{D}_{k}\right)+\sum_{i=1}^{n-k+j} c_{i} \widetilde{D}_{n-k+j-i}\left(\mathbf{D}_{k}\right) .
$$

Then:

$$
J_{k}(\mathrm{p})=\left(\widetilde{D}_{n-k+1}\left(\mathbf{D}_{k}, \mathrm{p}\right), \ldots, \widetilde{D}_{n}\left(\mathbf{D}_{k}, \mathrm{p}\right)\right) .
$$

Proof. Let $D_{t}^{\prime}=\sum_{i \geq 0} D_{i}^{\prime} t^{i}$ be the unique derivation on $\bigwedge M$ such that $D_{t}^{\prime} \nu^{j}=$ $\sum_{i \geq 0} \nu^{i+j} t^{i}$. Then

i) $D_{i}^{\prime} \in \mathcal{A}^{*}(\bigwedge M(\mathrm{p}))$ for each $i \geq 0$, 
ii) $\rho_{k}\left(D_{i}^{\prime}\right)=D_{i}$ if $1 \leq i \leq n-k$ and

iii) $\rho_{k}\left(D_{n-k+j}^{\prime}\right)=\widetilde{D}_{n-k+j}\left(\mathbf{D}_{k}, \mathrm{p}\right), \quad \forall j \geq 1$.

To check i), is sufficient to show that each $D_{i}^{\prime}$ is an $A$-polynomial expression in the $D_{i}$ s. As a matter of fact, if $i \leq n-k$ :

$$
\begin{aligned}
D_{i}^{\prime}\left(X^{1} \wedge \ldots \wedge X^{k}\right) & =D_{i}^{\prime}\left(\nu^{1} \wedge \ldots \wedge \nu^{k}\right)=\nu^{1} \wedge \ldots \wedge \nu^{k-1} \wedge \nu^{k+j}= \\
& =X^{1} \wedge \ldots \wedge X^{k-1} \wedge X^{k+i}=D_{i}\left(X^{1} \wedge \ldots \wedge X^{k}\right)
\end{aligned}
$$

and, for each $j \geq 1$ :

$$
\begin{gathered}
D_{n-k+j}^{\prime}\left(X^{1} \wedge \ldots \wedge X^{k}\right)=D_{n-k+j}^{\prime} \nu^{1} \wedge \ldots \wedge \nu^{k}=\nu^{1} \wedge \ldots \wedge \nu^{k-1} \wedge \nu^{n+j}= \\
=X^{1} \wedge \ldots \wedge X^{k-1} \wedge\left(X^{n+j}+c_{1} X^{n+j-1}+\ldots+c_{n+j-k} X^{k}+\ldots+c_{n} X^{j}\right)= \\
=X^{1} \wedge \ldots \wedge X^{k-1} \wedge X^{n+j}+\sum_{i=1}^{n-k+j} c_{i}\left(X^{1} \wedge \ldots \wedge X^{k-1} \wedge X^{n+j-i}\right)= \\
=\left(\tilde{D}_{n-k+j}\left(\mathbf{D}_{k}\right)+\sum_{i=1}^{n-k+j} c_{i} \tilde{D}_{n-k+j-i}\left(\mathbf{D}_{k}\right)\right) X^{1} \wedge \ldots \wedge X^{k} .
\end{gathered}
$$

Therefore formulas (19) and (20) show i), (19) shows ii) and (20) shows iii) above. We can now prove equality (17). Clearly $\left(\widetilde{D}_{n-k+j}\left(\mathbf{D}_{k}, \mathrm{p}\right)\right)_{j \geq 1} \subseteq J_{k}(\mathrm{p})$, because:

$$
\begin{aligned}
\widetilde{D}_{n-k+j}\left(\mathbf{D}_{k}, \mathrm{p}\right) \nu^{1} \wedge \ldots \wedge \nu^{k} & =D_{n-k+j}^{\prime} \nu^{1} \wedge \ldots \wedge \nu^{k}= \\
& =\nu^{1} \wedge \ldots \wedge \nu^{k-1} \wedge \nu^{n+j} \in \bigwedge^{k-1} M \wedge \mathrm{p} M .
\end{aligned}
$$

To show that $J_{k}(\mathrm{p}) \subseteq\left(\widetilde{D}_{n-k+j}\left(\mathbf{D}_{k}, \mathrm{p}\right)\right)_{j \geq 1}$ as well, let $P \in A\left[T_{1}, \ldots, T_{k}\right] \subseteq A[\mathbf{T}]$ such that $P\left(D^{\prime}\right) X^{1} \wedge \ldots \wedge X^{k} \in \wedge^{k-1} M \wedge \mathrm{p} M$. Without loss of generality one may assume that $P$ is homogeneous of degree $w$. Then

$$
P\left(D^{\prime}\right) \nu^{1} \wedge \ldots \wedge \nu^{k}=\sum a_{I} \Delta_{I}\left(D^{\prime}\right) \nu^{1} \wedge \ldots \wedge \nu^{k}=\sum \nu^{i_{1}} \wedge \ldots \wedge \nu^{i_{k}},
$$

where last sum is over all $\left(i_{1}, \ldots, i_{k}\right) \in \mathcal{I}^{k, w}$ such that $i_{k}>n$. By 2.9. $\Delta_{I}\left(D^{\prime}\right)$ belongs to the ideal $\left(D_{i_{k}-1}^{\prime}, \ldots, D_{i_{k}-k}^{\prime}\right)$ and, since $i_{k}>n$, one sees that if $\Delta_{I}\left(D^{\prime}\right) \nu^{1} \wedge \ldots \wedge \nu^{k} \in$ $\wedge^{k-1} M \wedge \mathrm{p} M$, then $\Delta_{I}\left(D^{\prime}\right) \in\left(\widetilde{D}_{n-k+j}\left(\mathbf{D}_{k}^{\prime}\right)\right)_{j \geq 1}$. The relation

$$
D_{n+1}^{\prime}-D_{n}^{\prime} \bar{D}_{1}^{\prime}+\ldots+(-1)^{n-k+1} D_{n-k+1}^{\prime} \bar{D}_{k}^{\prime}=0,
$$

holding in $\mathcal{A}^{*}\left(\bigwedge^{k} M(\mathrm{p})\right)$, implies that $D_{n+1}^{\prime}\left(\bigwedge^{k} M(\mathrm{p})\right) \in\left(D_{n-k+1}^{\prime}, \ldots, D_{n}^{\prime}\right) \bigwedge^{k} M(\mathrm{p})$ (here $(-1)^{i} \bar{D}_{i}^{\prime}$, as in 2.10, stands for the $i^{\text {th }}$ coefficient of $\left.\left(D_{t}^{\prime}\right)^{-1}\right)$.

By induction $D_{n+j}^{\prime}\left(\bigwedge^{k} M(\mathrm{p})\right) \in\left(D_{n-k+1}^{\prime}, \ldots, D_{n}^{\prime}\right) \bigwedge^{k} M$ as well. Because of (18), one hence has $J_{k}(\mathrm{p}) \subseteq\left(\rho_{k}\left(D_{n-k+1}^{\prime}\right), \ldots, \rho_{k}\left(D_{n}^{\prime}\right)\right)=\left(\widetilde{D}_{n-k+1}\left(\mathbf{D}_{k}, \mathrm{p}\right), \ldots, \widetilde{D}_{n}\left(\mathbf{D}_{k}, \mathrm{p}\right)\right)$, i.e. $J_{k}(\mathrm{p})$ is given precisely by (17).

4.8 Theorem. The following isomorphism holds:

$$
A^{*}\left(\bigwedge^{k} M(\mathrm{p})\right)=\frac{A\left[D_{1}, \ldots, D_{k}\right]}{\left(\widetilde{D}_{n-k+1}\left(\mathbf{D}_{k}, \mathrm{p}\right), \ldots, \widetilde{D}_{n}\left(\mathbf{D}_{k}, \mathrm{p}\right)\right)}
$$


Proof. Notation as in 4.6. Recall that by Corollary 4.5, formula (16), $D_{1}, \ldots, D_{k}$, are algebraically independent elements of $\mathcal{A}^{*}\left(\bigwedge^{k} M\right)$. Clearly, $P(D) \in \operatorname{ker}\left(\phi_{k}\right)$ if and only if $P(D) \epsilon^{1} \wedge \ldots \wedge \epsilon^{k} \in \bigwedge^{k-1} M \wedge \mathrm{p} M$, i.e. if and only if $P(D) \in J_{k}(\mathrm{p})$. Hence $\mathcal{A}^{*}\left(\bigwedge^{k} M(\mathrm{p})\right)=\mathcal{A}^{*}\left(\bigwedge^{k} M\right) / J_{k}(\mathrm{p})$, and the conclusion follows by Corollary 4.5 and Theorem 4.7

4.9 Remark. By [9, the polynomial $\mathrm{p}$, as above, splits in the ring $\mathcal{A}^{*}\left(\bigwedge^{k} M(\mathrm{p})\right)[X]$ as the product $\left(X^{k}+D_{1} X^{k-1}+\ldots+D_{k}\right) \cdot \mathrm{q}$, where $\mathrm{q}$ is a monic polynomial of degree $n-k$ (with $\mathcal{A}^{*}\left(\bigwedge^{k} M(\mathrm{p})\right)[X]$-coefficients).

4.10 Examples. Let $M=X A[X]$ and $\mathrm{p}(X)=X^{4}+c_{1} X^{3}+c_{2} X^{2}+c_{3} X+c_{4}, c_{i} \in A_{i}$. Then one has:

$$
\mathcal{A}^{*}\left(\bigwedge^{2} M(\mathrm{p})\right)=\frac{A\left[D_{1}, D_{2}\right]}{\left(\widetilde{D}_{3}\left(\mathbf{D}_{2}, \mathrm{p}\right), \widetilde{D}_{4}\left(\mathbf{D}_{2}, \mathrm{p}\right)\right)},
$$

where

$\widetilde{D}_{3}\left(\mathbf{D}_{2}, \mathbf{p}\right)=\widetilde{D}_{3}\left(\mathbf{D}_{2}\right)+c_{1} \widetilde{D}_{2}\left(\mathbf{D}_{2}\right)+c_{2} \widetilde{D}_{1}\left(\mathbf{D}_{2}\right)+c_{3}=2 D_{1} D_{2}-D_{1}^{3}+c_{1} D_{2}+c_{2} D_{1}+c_{3}$ and

$$
\begin{aligned}
\widetilde{D}_{4}\left(\mathbf{D}_{2}, \mathbf{p}\right) & =\widetilde{D}_{4}\left(\mathbf{D}_{2}\right)+c_{1} \widetilde{D}_{3}\left(\mathbf{D}_{2}\right)+c_{2} \widetilde{D}_{2}\left(\mathbf{D}_{2}\right)+c_{3} \widetilde{D}_{1}\left(\mathbf{D}_{2}\right)+c_{4}= \\
& =D_{2}^{2}+D_{1}^{2} D_{2}-D_{1}^{4}+c_{1}\left(2 D_{1} D_{2}-D_{1}^{3}\right)+c_{2} D_{2}+c_{3} D_{1}+c_{4},
\end{aligned}
$$

which we obtained from (13) and (14). Let us enumerate some particular cases.

1) If $A=\mathbb{Z}$, thought of as a graded ring concentrated in degree 0 , then $c_{i}=0,1 \leq i \leq 4$. Then $\mathrm{p}=X^{4}$ and presentation (22) becomes:

$$
\mathcal{A}^{*}\left(\bigwedge^{2} M\left(X^{4}\right)\right)=\frac{A\left[D_{1}, D_{2}\right]}{\left(2 D_{1} D_{2}-D_{1}^{3}, D_{2}^{2}+D_{1}^{2} D_{2}-D_{1}^{4}\right)},
$$

which coincides (Cf. 4, [5]) with the presentation of the integral cohomology ring of the grassmannian $G(2,4)$ of 2 -planes in $\mathbb{C}^{4}$ (or of the grassmannian of lines $G\left(1, \mathbb{P}^{3}\right.$ ) in the complex projective 3 -space).

2) If $A=\mathbb{Z}[q]$, and $\mathrm{p}(X)=X^{4}+q$, then (22) reads:

$$
\mathcal{A}^{*}\left(\bigwedge^{2} M\left(X^{4}+q\right)\right)=\frac{\mathbb{Z}[q]\left[D_{1}, D_{2}\right]}{\left(2 D_{1} D_{2}-D_{1}^{3}, D_{2}^{2}+D_{1}^{2} D_{2}-D_{1}^{4}+q\right)},
$$

which is the Witten-Siebert-Tian presentation of the small quantum cohomology ring $Q H^{*}(G(2,4))([14$, [13], [1]; see also [4);

3) If $\pi: E \rightarrow \mathcal{Y}$ is a holomorphic vector bundle of rank 4 on a smooth complex variety of dimension $m \geq 0$, and $\mathrm{p}(X)=X^{4}+\pi^{*} c_{1} X^{3}+\pi^{*} c_{2} X^{2}+\pi^{*} c_{3} X+\pi^{*} c_{4} \in A^{*}(\mathcal{Y})[X]$, where $c_{i}$ are the Chern classes of $E$ as in [2], p. 141, then $M(\mathrm{p})=A_{*}(\mathbb{P}(E)), A=A^{*}(\mathcal{Y})$ and $D_{1}=c_{1}\left(O_{\mathbb{P}(E)}(-1)\right)$, thought of as operator on $A_{*}(\mathcal{Y})$; in this case (22) gives the presentation of $A^{*}(G(2, E))$ (Cf. 3.11). If $\mathcal{Y}$ is a point, then $A^{*}(\mathcal{Y})=\mathbb{Z}, c_{i}=0$ and one recovers once again the presentation of the Chow ring of the grassmannian $G(2,4)$. 
4) Let $A=\mathbb{Z}\left[y_{1}, y_{2}, y_{3}, y_{4}\right]$ and $\mathrm{p}(X)=\prod_{i=1}^{4}\left(X-y_{i}+y_{1}\right)+q \in A[q]$. In this case presentation (22) is that the quantum equivariant cohomology ring $Q H_{T}^{*}(G(2,4))$ of the Grassmannian $G(2,4)$ under the action of a 4-dimensional compact or algebraic torus via a diagonal action with only isolated fixed points, as studied by Mihalcea in 11, Theorem 4.2, setting $p=2$ and $m=4$. This is compatible with the main result of the paper [6] (Theorem 3.7), with Theorem 2.9 of [4] and is now a consequence of [10. Notice that our generators are not the same as used in [11 (Cf. 6]).

\section{References}

[1] A. Bertram, Quantum Schubert Calculus, Adv. Math. 128, (1997) 289-305.

[2] W. Fulton, Intersection Theory, Springer-Verlag, 1984.

[3] W. Fulton, Equivariant Intersection Theory, Notes by Dave Anderson, Mich. Univ., 2005-2006 (http://www.math.lsa.umich.edu/ ${ }^{\sim}$ danderson/notes.html).

[4] L. Gatto, Schubert Calculus via Hasse-Schmidt Derivations, Asian J. Math., 9, No. 3, (2005), 315-322.

[5] L. Gatto, Schubert Calculus: an Algebraic Introduction, Instituto de Matemática Pura e Aplicada, Rio de Janeiro, 2005.

[6] L. Gatto, T. Santiago, Equivariant Schubert Calculus, Preprint Politecnico di Torino, n. 11, 2006, (http://calvino.polito.it/ gatto/preprints.htm).

[7] A. Knutson, T. Tao Puzzles and (equivariant) cohomology of Grassmannians, Duke Math. J. 119, no. 2 (2003), 221-260.

[8] D. Laksov, A. Thorup, A Determinantal Formula for the Exterior Powers of the Polynomial Ring, Indiana University Mathematics Journal, 2006, (to appear).

[9] D. Laksov, A. Thorup, Schubert Calculus on Grassmannians and Exterior Products, preprint, 2005.

[10] D. Laksov, The formalism of equivariant Schubert Calculus, Preprint, 2006.

[11] L. C. Mihalcea, Giambelli Formulae for the Equivariant Quantum Cohomology of the Grassmannian, Trans. Am. Math. Soc. (to appear) arXiv:math.CO/0506335 v2, 2004

[12] T. Santiago, Schubert Calculus on a Grassmann Algebra, Ph.D. Thesis, Politecnico di Torino, 2006.

[13] B. Siebert, G. Tian, On Quantum Cohomology rings of Fano manifolds and a formula of Vafa and Intrilligator, Asian J. Math 1 (1997), 679-695.

[14] E. Witten, The Verlinde Algebra and the cohomology of the Grassmannian, in "Geometry, Topology and Physics", Conference Proceedings and Lecture Notes in Geometric Topology, Vol. IV, pp. 357-422, International Press, Cambridge, MA, 1995.

Dipartimento di Matematica, Politecnico di Torino, C.so Duca degli Abruzzi, 24, 10129, Torino

letterio.gatto@polito.it

taise@calvino.polito.it 